DOI: $10.32326 / 1814-9146-2019-81-3-271-291$

НЕМОНОТОННОСТЬ, ЗНАКОПЕРЕМЕННОСТЬ И ДРУГИЕ ОСОБЕННОСТИ ПОВЕДЕНИЯ КОЭФФИЦИЕНТА ПУАССОНА ЛИНЕЙНО ВЯЗКОУПРУГИХ МАТЕРИАЛОВ ПРИ РАСТЯЖЕНИИ С ПОСТОЯННОЙ СКОРОСТЬЮ*

(C) 2019 г.

Хохлов А.В.

НИИ механики Московского государственного университета имени М.В. Ломоносова, Москва, Российская Федерация

andrey-khokhlov@ya.ru

Поступила в редакичию 31.01.2019

Аналитически исследуется способность линейного интегрального определяющего соотношения вязкоупругости Больцмана - Вольтерры для изотропных материалов описывать различные эффекты эволюции трехмерного деформированного состояния и возможные типы поведения коэффициента поперечной деформации (коэффициента Пуассона), наблюдаемые в одноосных испытаниях реономных материалов на растяжение (сжатие) с постоянной скоростью и на ползучесть, в частности эффекты возрастания, убывания, немонотонности, знакопременности и отрицательности коэффициента Пуассона. Это соотношение не учитывает влияние шаровой и девиаторной частей тензоров напряжений и деформаций друг на друга и содержит две произвольные материальные функции одного аргумента (функции объемной и сдвиговой ползучести).

При произвольных функциях ползучести (на них наложены минимальные необходимые ограничения: они предполагаются положительными, возрастающими и выпуклыми вверх на полуоси времени) проанализированы выражения для коэффициента Пуассона и параметра вида деформированного состояния (равного отношению объемной деформации к интенсивности деформаций) через функции ползучести, скорость нагружения и время, изучены общие качественные свойства и характерные особенности зависимости коэффициента Пуассона от времени. Получена точная универсальная двусторонняя оценка для диапазона изменения коэффициента Пуассона, доказаны критерии его монотонности и немонотонности и критерий его отрицательности на некотором интервале времени в зависимости от качественных свойств функций объемной и сдвиговой ползучести. Обнаруженные свойства сопоставлены со свойствами коэффициента Пуассона в условиях ползучести (при постоянном напряжении) и проиллюстрированы на конкретных примерах моделей с классическими функциями ползучести и фрактальных моделей. Анализ показал, что линейная теория способна качественно воспроизводить основные эффекты, связанные с поведением коэффициента Пуассона (монотонность, немонотонность, знакопеременность, отрицательность на некотором интер-

* Выполнено при поддержке РФФИ (грант № 17-08-01146_a). 
вале времени, стабилизацию с течением времени), за исключением зависимости от скорости нагружения.

Ключевые слова: вязкоупругость, объемная ползучесть, нагружение с постоянной скоростью, коэффициент поперечной деформации, немонотонность и знакопеременность коэффициента Пуассона, развитие эффекта ауксетичности во времени, вязкоупругие ауксетики, магнитные композиты, индикаторы областей линейности и нелинейности.

\section{Введение}

При одноосном растяжении (сжатии) образца любого материала, как известно, возникают не только осевые деформации $\varepsilon_{\|,}$, но и поперечные $\varepsilon_{\perp}$. У линейно упругих материалов отношение $v=-\varepsilon_{\perp} / \varepsilon_{\|}$не зависит от величины растягивающей силы, времени и предыстории деформирования и называется коэффициентом поперечной деформации (КПД), или коэффициентом Пуассона (в направлении оси нагружения). У изотропных материалов $\mathrm{V}$ - материальная постоянная, которая с модулем Юнга полностью определяет тензор модулей упругости, в частности, связь объемной деформации $\theta$ со средним напряжением.

Коэффициент Пуассона изотропных вязкоупругопластичных материалов при одноосном нагружении (даже постоянной нагрузкой) зависит от времени (от продольной деформации $\left.\varepsilon_{\|}(t)\right)$ и от истории (программы) нагружения, то есть не является материальной функцией, хотя и остается важной характеристикой. Зависимости объемной и поперечной деформаций от времени и $\varepsilon_{\|}$, характер изменения и диапазоны значений КПД для реономных материалов (полимеров, твердых топлив, асфальтобетонов, ударопрочных полистиролов, АБС-пластиков и других дисперсно наполненных полимеров, прессованных порошковых композитов, сплавов, керамик, металлических и полимерных пен, льдов, грунтов, горных пород и т.п.) весьма разнообразны даже при малых деформациях, даже в стандартных испытаниях на релаксацию, ползучесть при постоянной нагрузке или нагружение с постоянной скоростью [1-45]. У большинства металлов, многих стекол, полимеров (например, полиэтиленов высокой плотности, ПММА, эпоксидных смол и т.п.) и порошковых композитов наблюдается монотонное возрастание $v(t)$ с ростом $\varepsilon_{\|}(t)[5,6,9,13,17$, $18,20,23,24,37]$. У многих реономных материалов, как достаточно хрупких, так и высокоэластичных (твердое топливо, асфальтобетон, АБС-пластики, чугун и т.п.), наблюдается убывание $v(t)$, вызванное, как обычно считается, необратимым изменением объема при растяжении или сжатии $[1,3,4,8,10,16,19,20,25]$. У некоторых материалов объемная деформация и КПД меняются немонотонно и меняют знак [3, $4,7,20,22,32,42]$. В последние три десятилетия обнаружены, активно конструируются, исследуются и синтезируются новые материалы и конструкции (в частности, нанотрубки и напечатанные ячеистые структуры) с отрицательными КПД в некоторых направлениях (auxetics) [26-39]; в подавляющем большинстве статей изучаются упругие (как правило, анизотропные) частичные огзетики (прижилась транслитерация «ауксетики»), особенности эффекта смены знака и эволюции отрицательного КПД реономных материалов во времени не анализировались в общей постановке. В ряде публикаций исследуется влияние на КПД вязкоупругих материалов программы одноосного нагружения [11-15, 17, 22, 23, 40-44], в частности зависимость КПД от скорости нагружения, а также влияние на кривые деформирования и ползучести и на эволюцию коэффициента Пуассона наложения всестороннего 
давления на одноосное растяжение или сдвиг [1-4, 10, 19, 45-48].

Изменение КПД и связанные с ним типичные механические эффекты эволюции трехмерного деформированного состояния при заданном одноосном напряженном состоянии рабочей части образца следует учитывать при обработке и интерпретации кривых испытаний наследственных материалов (в частности методами индентирования) и при выборе и идентификации определяющего соотношения (ОС) для моделирования их поведения. Для выбора того или иного ОС и его совершенствования важно знать, какие механические эффекты оно способно моделировать и при каких требованиях к материальным функциям, в частности, какие из упомянутых эффектов, связанных с эволюцией поперечной деформации и КПД. Для этого необходимо системное аналитическое исследование общих свойств кривых деформирования, ползучести и релаксации, порождаемых ОС с произвольными материальными функциями при разных типовых программах нагружения, и их зависимости от параметров программ нагружения и характеристик материальных функций. В частности, необходим системный анализ возможностей линейного ОС Больцмана - Вольтерры:

$$
\begin{gathered}
\varepsilon_{i j}(t)=e_{i j}+\varepsilon_{0} \delta_{i j}, \quad e_{i j}=\frac{3}{2} \Pi s_{i j}(t), \quad \theta=\Pi_{0} \sigma_{0}, \\
\sigma_{0}(t)=\frac{\sigma_{i i}(t)}{3}, \quad s_{i j}=\sigma_{i j}-\sigma_{0} \delta_{i j}, \quad \theta(t)=3 \varepsilon_{0}=\varepsilon_{i i}(t), \\
\Pi y=\int_{0}^{t} \Pi(t-\tau) d y(\tau), \quad \Pi_{0} y=\int_{0}^{t} \Pi_{0}(t-\tau) d y(\tau), \quad t>0,
\end{gathered}
$$

с двумя материальными функциями (сдвиговой и объемной ползучести) $\Pi(t)$ и $\Pi_{0}(t)$ $[2,49,50]$, изучение качественных свойств порождаемых им базовых теоретических кривых, которые следуют из постулатов о наследственности, линейности и инвариантности относительно сдвигов по времени операторов (2), связывающих истории изменения компонент девиаторов $\mathbf{s}=\boldsymbol{\sigma}-\sigma_{0} \mathbf{I}, \mathbf{e}=\boldsymbol{\varepsilon}-\varepsilon_{0} \mathbf{I}$ и шаровых частей тензора напряжений $\boldsymbol{\sigma}(t)$ и тензора малых деформаций $\boldsymbol{\varepsilon}(t)$ в точке тела, и постулата об отсутствии перекрестного влияния шаровых и девиаторных частей тензоров $\boldsymbol{\sigma}(t)$ и $\boldsymbol{\varepsilon}(t)$ друг на друга. Это весьма полезно для контроля и адекватной интерпретации данных испытаний и для выбора или построения более сложных и точных нелинейных моделей поведения реономных материалов, опирающихся на линейную теорию наследственности и обобщающих ее в определенных аспектах, для их идентификации, аттестации и сопоставления. Ведь линейное ОС (1) играет роль своеобразного «окуляра» для наблюдения и отсчетной базы для сопоставления и идентификации, по отношению к которой естественно трактовать и изучать эффекты нелинейного поведения материалов (отклонения от предсказаний линейной модели как начального приближения), наблюдаемые в испытаниях материалов. В частности, такой анализ представляется полезным для оценки данных испытаний и выводов разных исследователей о поведении КПД «одного и того же» материала (в «одинаковых» условиях испытаний), нередко противоречащих друг другу из-за сложностей реализации достаточно точных и длительных измерений и качественной обработки результатов и, возможно, особенностей теоретических представлений авторов.

Настоящая статья продолжает цикл работ [48, 51-58] по системному исследованию комплекса моделируемых и немоделируемых реологических эффектов, сфер 
влияния материальных функций и границ области применимости линейного ОС (1) и физически нелинейного ОС

$$
\begin{gathered}
\varepsilon_{i j}(t)=\frac{3}{2} \Phi(L(t)) \sigma^{-1}(t)\left[\sigma_{i j}-\sigma_{0} \delta_{i j}\right]+\frac{1}{3} \Phi_{0}\left(L_{0}(t)\right) \delta_{i j}, \\
L(t)=\Pi \sigma, \quad L_{0}(t)=\Pi_{0} \sigma_{0},
\end{gathered}
$$

с четырьмя произвольными материальными функциями $\Pi(t), \Phi(x), \Pi_{0}(t), \Phi_{0}(x)$. OC (3) - один из вариантов распространения на трехосный случай нелинейного уравнения наследственности $\varphi(\varepsilon(t))=\int_{0}^{t} \Pi(t-\tau) d \sigma(\tau)$, предложенного Ю.Н. Работновым $[59,60]$ в качестве обобщения одноосного линейного ОС (1) путем введения второй материальной функции $\varphi(u)$ (подробную библиографию по этим темам см. в $[48,51-58])$. ОС (1) и (3) описывают процессы изотермического деформирования нестареющих изотропных вязкоупругих сред; они связывают истории изменения тензоров (малых) деформаций $\boldsymbol{\varepsilon}(t)$ и напряжений $\boldsymbol{\sigma}(t)$ в произвольной точке тела в предположении отсутствия взаимного влияния шаровых и девиаторных частей тензоров е и $\mathbf{s}$, то есть при независимости объемной деформации $\theta(t)$ от касательных напряжений, а сдвиговых деформаций - от среднего напряжения $\sigma_{0}(t)$ и пренебрежении влиянием третьих инвариантов тензоров $[2,49,50]$. Множитель $3 / 2$ вынесен из функции ползучести (ФП) П $(t)$ в (1) для удобства сопоставления с результатами анализа нелинейного ОС (3) с $\Phi(x)=x$.

Цели настоящей статьи:

- анализ общих качественных свойств зависимости КПД (коэффициента Пуассона) от времени, порождаемой ОС (1) с произвольными ФП П $(t), \Pi_{0}(t)$ при одноосном нагружении с постоянной скоростью

$$
\sigma_{11}(t)=b t \mathrm{~h}(t), \quad \sigma_{i j}(t)=0 \text { при } i+j>2,
$$

где $b \neq 0$ - скорость нагружения, $\mathrm{h}(t)$ - функция Хевисайда;

- вывод критериев монотонности и немонотонности КПД и критерия его отрицательности на некотором интервале времени в зависимости от качественных свойств функций объемной и сдвиговой ползучести.

Время и компоненты тензора напряжений предполагаются безразмерными. В силу линейности ОС (1) изучаемые качественные свойства кривых деформирования и КПД не зависят от способа масштабирования напряжений и времени.

\section{1. Минимальные ограничения на функции ползучести линейного ОС (1)}

Обращение ОС (1), как известно [2, 49, 50], имеет вид

$$
\sigma_{0}=\mathbf{R}_{0} \theta, \quad s_{i j}=\frac{2}{3} \mathbf{R} e_{i j}, \quad \mathbf{R} y=\int_{0}^{t} R(t-\tau) d y(\tau), \quad \mathbf{R}_{0} y=\int_{0}^{t} R_{0}(t-\tau) d y(\tau), t>0,
$$

где функции релаксации $R(t)$ и $R_{0}(t)$ связаны с ФП П и П интегральными уравнениями

$$
\int_{0}^{t} R(t-\tau) \Pi(\tau) d \tau=t, \quad \int_{0}^{t} R_{0}(t-\tau) \Pi_{0}(\tau) d \tau=t, t>0,
$$

выражающими условия взаимной обратности операторов $\Pi \mathbf{R}=\mathbf{R} \Pi=\mathbf{I}$ и $\boldsymbol{\Pi}_{0} \mathbf{R}_{0}=$ $=\mathbf{R}_{0} \boldsymbol{\Pi}_{0}=\mathbf{I}$. 
Функции ползучести и релаксации $П(t), \Pi_{0}(t), R(t), R_{0}(t)$ в ОС (1), (5) предполагаются положительными и дифференцируемыми на $(0, \infty)$, функции П и $\Pi_{0}-$ возрастающими и выпуклыми вверх [51-54], а $R$ и $R_{0}$ - убывающими и выпуклыми вниз на $(0, \infty) ; R$ и $R_{0}$ могут иметь интегрируемую особенность или $\delta$-сингулярность в точке $t=0$ (слагаемое $\eta \delta(t), \eta>0, \delta(t)$ - дельта-функция). Из этих условий следует, в частности, существование пределов $R(+\infty)=\inf R(t) \geq 0, R(0)=\sup R(t)>$ $>0(y(0)=y(0+)$ - обозначение для предела функции $y(t)$ справа в точке $t=0$; $R(0)=+\infty$, если $R(t)$ не ограничена сверху) и $\Pi(0)=\inf \Pi(t) \geq 0$.

Если $\Pi(0) \neq 0$ и $\Pi_{0}(0) \neq 0$ (такие модели будем называть регулярными), то $R(0)=1 / \Pi(0)<\infty$ и $R_{0}(0)=1 / \Pi_{0}(0)<\infty$ (то есть мгновенный модуль сдвига $2 G=$ $=(2 / 3) R(0)$ и объемный модуль $K=R_{0}(0)$ конечны) и на линейном пространстве неnрерывных кусочно-гладких при $t \geq 0$ функций операторы ОС (2) и (5) представимы в виде операторов Вольтерры второго рода:

$$
\boldsymbol{\Pi} y=\Pi(0) y(t)+\int_{0}^{t} \dot{\Pi}(t-\tau) y(\tau) d \tau, \quad \mathbf{R} y=R(0) y(t)+\int_{0}^{t} \dot{R}(t-\tau) y(\tau) d \tau, \quad t \geq 0 .
$$

Все структурные реологические модели из линейных пружин и демпферов описываются ОС (1). Например, семейство функций ползучести

$$
\Pi(t)=\alpha t+\beta-\gamma \exp (-\lambda t), \quad \lambda>0, \alpha, \beta \geq 0, \gamma \in[0, \beta],
$$

удовлетворяет всем требованиям к ФП и в случае $\gamma \in(0, \beta), \alpha, \beta>0$, порождает все четыре структурно различные (но эквивалентные [52]) четырехзвенные модели из двух пружин и двух демпферов (они регулярны, $R=E_{1} \exp \left(-\mu_{1} t\right)+E_{2} \exp \left(-\mu_{2} t\right)$ и $R(+\infty)=0)$, а при $\alpha=0$ - трехзвенные модели Кельвина и Пойнтинга-Томпсона с одним демпфером (они регулярны и эквивалентны, $R=E \exp (-\mu t)+r$ и $R(+\infty)=r>0$ ). Поскольку П $(0)=\beta-\gamma$, то семейство (7) порождает нерегулярные модели лишь в случае $\gamma=\beta$ : при $\lambda \beta=0$ - ньютоновскую жидкость $(R=\eta \delta(t))$, при $\alpha=0$ - модель Фойгта $(R=\eta \delta(t)+r)$, при $\alpha>0$ - обе трехзвенные модели с одной пружиной и двумя демпферами $(R=\eta \delta(t)+E \exp (-\mu t), R(+\infty)=0)$. При $\gamma=0$ (7) дает модель Максвелла $(R=E \exp (-\mu t))$.

\section{2. Свойства коэфффициента Пуассона при растяжении с постоянной скоростью}

Для одноосного нагружения вида (4) $\sigma_{0}=\sigma_{11} / 3=(1 / 3) b t \mathrm{~h}(t)$ (функцию Хевисайда $\mathrm{h}(t)$ в дальнейшем будем опускать, полагая, что $t>0)$, а девиатор напряжений - диагональный тензор $\mathbf{s}(t)=(1 / 3) b t \operatorname{diag}(2,-1,-1)$. Из (1) следует, что девиатор деформаций тоже диагонален: $\mathbf{e}=0,5 b Q(t) \operatorname{diag}(2,-1,-1)$, а объемная деформация

$$
\begin{gathered}
\theta(t, b)=\Pi_{0} \sigma_{0}=\frac{1}{3} b Q_{0}(t), \quad t>0, \\
Q(t)=\int_{0}^{t} \Pi(\tau) d \tau, \quad Q_{0}(t)=\int_{0}^{t} \Pi_{0}(\tau) d \tau .
\end{gathered}
$$

У тензора деформаций $\boldsymbol{\varepsilon}=\mathbf{e}+(1 / 3) \theta \mathbf{I}$ тоже отличны от нуля только диагональные элементы:

$$
\varepsilon_{11}(t, b)=b Q(t)+\frac{1}{9} b Q_{0}(t)
$$




$$
\varepsilon_{22}(t, b)=\varepsilon_{33}(t, b)=-\frac{1}{2} b Q(t)+\frac{1}{9} b Q_{0}(t) .
$$

Интенсивности напряжений и деформаций:

$$
\sigma(t)=\left(\frac{3}{2} s_{i j} s_{i j}\right)^{0,5}=|b| t=3\left|\sigma_{0}\right|, \quad \varepsilon=\left(\frac{2}{3} e_{i j} e_{i j}\right)^{0,5}=|b| Q(t) .
$$

Для любой скорости $b>0$ объемная и осевая деформации (8) и (9) и интенсивность деформаций - положсительные, возрастающие и выпуклые вниз функиии времени, поскольку

$$
\begin{gathered}
\dot{\theta}=\frac{1}{3} b \Pi_{0}(t)>0, \quad \ddot{\theta}=\frac{1}{3} b \dot{\Pi}_{0}(t)>0, \\
\dot{\varepsilon}_{11}=b \Pi(t)+\frac{1}{9} b \Pi_{0}(t)>0, \quad \ddot{\varepsilon}_{11}=b \dot{\Pi}_{0}(t)+\frac{1}{9} b \dot{\Pi}_{0}(t)>0
\end{gathered}
$$

(в силу положительности и возрастания ФП). Поперечная деформация $\varepsilon_{\perp}=\varepsilon_{22}(t)$ (10) не обязана быть ни монотонной, ни выпуклой функцией: она может убывать или возрастать на всем интервале $t>0$, может иметь точки экстремума и перегиба и менять знак. Так как

$$
\dot{\varepsilon}_{\perp}=-\frac{1}{2} b \dot{Q}+\frac{1}{9} b \dot{Q}_{0}=-\frac{1}{2} b \Pi(t)+\frac{1}{9} b \Pi_{0}(t), \quad \ddot{\varepsilon}_{\perp}=-\frac{1}{2} b \dot{\Pi}(t)+\frac{1}{9} b \dot{\Pi}_{0}(t),
$$

то при $b>0$ критерии (нестрогого) возрастания и выпуклости вниз $\varepsilon_{\perp}(t)$ на некотором интервале времени имеют вид $\Pi_{0}(t) \geq 4,5 \Pi(t)$ или $\dot{\Pi}_{0}(t) \geq 4,5 \check{\Pi}(t)$, а уравнения для точек экстремума и перегиба $-\Pi_{0}(t)=4,5 \Pi(t)$ или $\dot{\Pi}_{0}(t)=4,5 \dot{\Pi}(t)$.

Например, в случае модели с $\Pi_{0}(t)=c=$ const $>0$ и произвольной ФП П, то есть когда зависимость объемной деформации от среднего напряжения считается упругой (эта гипотеза часто применяется для упрощения решения краевых задач), имеем $Q_{0}=c t, \theta=(1 / 3) b c t$,

$$
\varepsilon_{\perp}=-\frac{1}{2} b Q(t)+\frac{1}{9} b c t, \quad \dot{\varepsilon}_{\perp}=-\frac{1}{2} b \Pi(t)+\frac{1}{9} b c, \quad \ddot{\varepsilon}_{\perp}=-\frac{1}{2} b \dot{\Pi}(t)<0,
$$

то есть $\varepsilon_{\perp}(t)$ выпукла вверх, но может не быть монотонной. Уравнение для точек экстремума имеет вид $\Pi(t)=(2 / 9) c$, поэтому в случае $\Pi(0)<(2 / 9) c<\Pi(\infty) \varepsilon_{\perp}(t)$ имеет (единственную) точку максимума и положительна в некоторой правой окрестности нуля; если $c \leq 4,5 \Pi(0)$, то $\varepsilon_{\perp}(t)$ убывает на луче $t \geq 0$, а если $c \geq 4,5 \Pi(\infty)-$ возрастает. Отметим, что при ползучести эта модель (с произвольной ФП П) всегда порождает монотонную поперечную деформацию $\varepsilon_{\perp}(t)$ [54].

Поделив (10) на (9), найдем КПД при нагружении (4):

или

$$
v(t)=-\frac{\varepsilon_{\perp}}{\varepsilon_{11}}=\frac{1}{2} \frac{9 b Q(t)-2 b Q_{0}(t)}{9 b Q(t)+b Q_{0}(t)}=\frac{1}{2}-\frac{3 Q_{0}(t)}{18 Q(t)+2 Q_{0}(t)},
$$

$$
\begin{aligned}
v(t)=f(z \xi), \quad f(x) & =0,5-3 x(6+2 x)^{-1}=-1+9(6+2 x)^{-1}, \\
\xi(t) & =3 \frac{\varepsilon_{0}}{\varepsilon}=\frac{\theta}{\varepsilon}=\frac{1}{3} \frac{z Q_{0}(t)}{Q(t)} .
\end{aligned}
$$

Здесь $\xi$ - параметр вида деформированного состояния, $z=\operatorname{sgn} b= \pm 1, \varepsilon=$ $=\left((2 / 3) e_{i j} e_{i j}\right)^{0,5}=|b| Q(t)-$ интенсивность деформаций. Аналогичный параметр вида 
напряженного состояния $\xi_{\sigma}=3 \sigma_{0}(t) / \sigma(t)=b t /(|b| t)=z$ не зависит от времени и от скорости.

Существенное отличие линейного ОС (1) от нелинейных ОС вязкоупругости независимость КПД и параметра (15) от скорости нагружения и ее знака. Это свойство, если оно не выполняется в испытаниях некоторого материала на нагружение с разными скоростями, можно использовать как индикатор нелинейности его поведения и неприменимости линейного ОС (1). Если же оно выполняется (см., например, [11]), то это аргумент в пользу гипотезы о линейно вязкоупругом поведении материала (не осознанный в [11]).

Так как $Q(t)>0$ и $Q_{0}(t)>0$ при $t>0$, то $\theta \geq 0, \xi \geq 0$ и $v(t) \leq 0,5$. Из $Q(t)>0$ (то есть из $\Pi(t)>0)$ следует оценка снизу $v(t)>-1$. Таким образом, для любых ФП в ОС (1) верна оценка

$$
-1<v(t)<0,5, \quad t>0
$$

Покажем, что эта оценка точна. Так как $\dot{Q}=\Pi$ и $\dot{Q}_{0}=\Pi_{0}$, то по правилу Лопиталя пределы $\xi(0+)$ и $\xi(\infty)$ при $t \rightarrow 0+$ и $t \rightarrow+\infty$ совпадают с пределами отношения $P=(1 / 3) z \Pi_{0}(t) / \Pi(t)$ :

$$
\xi(0+)=P(0), \quad \xi(\infty)=P(\infty), \quad v(0+)=f(P(0)), \quad v(\infty)=f(P(\infty)) .
$$

Поэтому для моделей с $\Pi_{0}(0)=0$ (объемно нерегулярных) и П $(0) \neq 0$ формула $(15)$ в пределе при $t \rightarrow 0+$ дает $\xi(0+)=0$ и по (14) $\mathrm{v}(0+)=0,5$ для любого $b>0$, а для моделей с $\Pi(0)=0$ и $\Pi_{0}(0) \neq 0$ имеем $\xi(0+)=\infty$ и по (14) $v(0+)=-1$.

Из (17) следует, что функция $v(t)$ всегда имеет горизонтальную асимптоту при $t \rightarrow \infty$, поскольку предел $v(\infty)$ (равновесное значение КПД) всегда конечен: $v(\infty)=$ $=-1$, если $P(\infty)=\infty$, и $\vee(\infty) \in(-1 ; 0,5]$, если $P(\infty)<\infty$. Кроме того, начальное $u$ равновесное значения КПД при нагружении (4) такие же, как в условиях ползучести при постоянном напряжении [54].

КПД (13) при нагружении (4) может быть отрицательным, поскольку возможно $\varepsilon_{\perp}(t)>0$. Критерий отрицательности $v(t)$ на некотором интервале времени при растяжении имеет вид

$$
Q_{0}(t)>\frac{9}{2} Q(t)
$$

В зависимости от конкретных свойств ФП (в зависимости от структуры множества $Z=\left\{t \mid t>0, Q_{0}(t)=4,5 Q(t)\right\}$ нулей функции $\left.\varepsilon_{\perp}(t)\right)$ эта область отрицательности КПД может быть пустой, может совпадать с полуосью $t>0$, а может состоять из нескольких компонент связности (интервалов оси времени). Если $\Pi(\infty)<\infty$ и $\Pi_{0}(\infty)>$ $>4,5 \Pi(\infty)$, то область (18) содержит луч $t>t_{*}$, где $t_{*}=\sup Z<\infty$. Если $\Pi_{0}(0)>4,5 \Pi(0)$, то область (18) содержит правую окрестность нуля $\left(0, t_{0}\right)$, где $t_{0}=\inf Z>0$; в этом случае $\left(0, t_{0}\right)$ шире, чем первая компонента связности $\left(0, t_{c}\right)$ области отрицательности КПД при ползучести, задаваемой неравенством $\Pi_{0}(t)>4,5 \Pi(t)$ [54], ибо из справедливости последнего неравенства на $\left(0, t_{c}\right)$ и непрерывности ФП следует, что и неравенство (18) для интегралов от ФП выполняется при всех $t \leq t_{c}$.

Из (13) следует, что КПД $v(t)$, вообще говоря, не постоянен. Критерий независимости КПД от времени при растяжении с постоянной скоростью (то есть критерий постоянства $\xi(t)=k, k>0$, в силу (14)) налагает связь на сдвиговую и объемную ФП ОС (1): $Q_{0}(t)=3 k Q(t)$ при $t>0$, то есть 


$$
\Pi_{0}(t)=3 k \Pi(t), \quad t>0 .
$$

В силу (14) $k=3(0,5-v) /(1+v)$. Тождество (19) обеспечивает в силу ОС (1) постоянство КПД при одноосном нагружении по любой программе $\sigma_{11}(t)$. В частности, тождество (19) выполняется для несжимаемого материала $\left(\mathrm{c} \Pi_{0}(t) \equiv 0\right)$, когда $v(t) \equiv$ $\equiv 0,5$ по (13).

КПД (13) при нагружении (4) не обязан быть монотонной функцией. Поскольку из (14) следует, что

$$
\dot{v}(t)=-3(6+2 \xi)^{-2}[\dot{\xi}(6+2 \xi)-2 \dot{\xi} \xi]=-18(6+2 \xi)^{-2} \dot{\xi},
$$

то знаки $\dot{v}(t)$ и $-\dot{\xi}(t)$ одинаковы и интервалы монотонности $v(t)$ и $-\xi(t)$ совпадают. Так как дифференцирование функции (15) дает

$$
\dot{\xi}(t)=\frac{1}{3} Q^{-2}(t) y(t), \quad y(t)=\Pi_{0}(t) Q(t)-Q_{0}(t) \Pi(t),
$$

то критерий (нестрогого) возрастания КПД (убывания $\xi(t))$ на некотором интервале имеет вид $y(t) \leq 0$, то есть $\Pi_{0}(t) / Q_{0}(t) \leq \Pi(t) / Q(t)$, а необходимое условие экстремума - равенство $y(t)=0$, то есть

$$
\frac{\Pi_{0}(t)}{Q_{0}(t)}=\frac{\Pi(t)}{Q(t)}
$$

Если равенство (22) выполняется на некотором интервале времени (не обязательно совпадающем с полуосью $t>0)$, то $\dot{\mathrm{v}}(t)=0$ и $v(t)=$ const на этом интервале.

Пр и мер 1. Рассмотрим модель (1) с (ограниченными) ФП классической модели Кельвина

$$
\begin{gathered}
\Pi=\beta-\gamma \exp (-\lambda t), \quad \Pi_{0}=\beta_{0}-\gamma_{0} \exp \left(-\lambda_{0} t\right), \\
\lambda, \beta, \lambda_{0}, \beta_{0}>0, \quad \gamma \in(0, \beta), \gamma_{0} \in\left(0, \beta_{0}\right) .
\end{gathered}
$$

Смысл параметров: $\tau=1 / \lambda$ и $\tau_{0}=1 / \lambda_{0}-$ времена ретардации при сдвиге и изменении объема,

$$
\begin{gathered}
G=\frac{1}{2} \cdot \frac{2}{3} \Pi^{-1}(0)=\frac{1}{3}(\beta-\gamma)^{-1}, \quad G_{\infty}=\frac{1}{2} \cdot \frac{2}{3} \Pi^{-1}(\infty)=\frac{1}{3} \beta^{-1}, \\
K=\frac{1}{\Pi_{0}(0)}=\frac{1}{\beta_{0}-\gamma_{0}}, \quad K_{\infty}=\frac{1}{\Pi_{0}(\infty)}=\frac{1}{\beta_{0}}
\end{gathered}
$$

- мгновенный и длительный модули сдвига и объемные модули. Для ФП (23) имеем

$$
Q=\beta t-\gamma \lambda^{-1}(1-\exp (-\lambda t)), \quad Q_{0}=\beta_{0} t-\gamma_{0} \lambda_{0}^{-1}\left(1-\exp \left(-\lambda_{0} t\right)\right),
$$

и согласно (17)

$$
\begin{gathered}
\xi(0)=\frac{\beta_{0}-\gamma_{0}}{3(\beta-\gamma)}=\frac{G}{K}, \quad v(0)=-1+\frac{27(\beta-\gamma)}{18(\beta-\gamma)+2\left(\beta_{0}-\gamma_{0}\right)}, \\
\xi(\infty)=\frac{\beta_{0}}{3 \beta}=\frac{G_{\infty}}{K_{\infty}}, \quad v(\infty)=-1+\frac{27 \beta}{18 \beta+2 \beta_{0}} .
\end{gathered}
$$

При $t \rightarrow \infty$ функции $\xi(t)$ и $v(t)$ имеют горизонтальные асимптоты, не зависящие от $\lambda, \lambda_{0}, \gamma$ и $\gamma_{0}$. Согласно (21) 


$$
\begin{aligned}
y & =\left(\beta_{0}-\gamma_{0} \exp \left(-\lambda_{0} t\right)\right)\left[\beta t-\gamma \lambda^{-1}(1-\exp (-\lambda t))\right]- \\
& -\left[\beta_{0} t-\gamma_{0} \lambda_{0}^{-1}\left(1-\exp \left(-\lambda_{0} t\right)\right)\right](\beta-\gamma \exp (-\lambda t)) .
\end{aligned}
$$

При $\lambda=\lambda_{0}$ можно привести $y(t)$ к виду $y=\left(\beta \gamma_{0}-\beta_{0} \gamma\right)\left[\lambda^{-1}-\left(\lambda^{-1}+t\right) \exp (-\lambda t)\right]$. Так как $\dot{y}=\left(\beta \gamma_{0}-\beta_{0} \gamma\right) \lambda t \exp (-\lambda t)$ и $y(0)=0$, то при $\gamma_{0} \beta>\gamma \beta_{0} y(t)$ возрастает и $y(t)>0$ при $t>0$, а при $\gamma_{0} \beta<\gamma \beta_{0} y(t)$ убывает и $y(t)<0$. Поэтому в силу (21) при $\gamma_{0} / \gamma>\beta_{0} / \beta$ $\xi(t)$ возрастает, а КПД (13) убывает на полуоси $t>0$, при $\gamma_{0} / \gamma<\beta_{0} / \beta \xi(t)$ убывает, а КПД возрастает при $\mathrm{t}>0$ (в точности, как и КПД модели (23) при ползучести [54]). При $\gamma_{0} / \gamma=\beta_{0} / \beta y(t) \equiv 0, \xi(t)=G / K=G_{\infty} / \mathrm{K}_{\infty}=k$ и $v(t)=-1+9(6+2 \mathrm{k})^{-1}$, то есть КПД постоянен и выполнено тождество (19). Условие отрицательности КПД (18) имеет при $\lambda=\lambda_{0}$ вид $\beta_{0} t-\gamma_{0} \lambda^{-1}(1-\exp (-\lambda t))>4,5\left(\beta t-\gamma \lambda^{-1}(1-\exp (-\lambda t))\right.$, то есть $\left(\gamma_{0}-4,5 \gamma\right) /(\lambda t)<\beta_{0}-4,5 \beta$; в частности, если $\beta_{0}>4,5 \beta$, а $\gamma_{0}>4,5 \gamma$, то оно выполнено при всех $t>0$ и $\lambda>0$. При $\lambda \neq \lambda_{0}$ функция $y(t)$ может менять знак, и потому $\xi(t)$ и $v(t)$ могут иметь точки экстремума.

На рис. $1 a$ приведены графики КПД $v(t)$ при нагружении (4) трех моделей вида (23) с одинаковыми сдвиговыми ФП П $(t)(\lambda=0,1, \beta=1, \gamma=0,5$ и $\tau=1 / \lambda=10)$ и разными $\Pi_{0}(t): \mathrm{c} \lambda_{0}=\lambda=0,1$ (голубые кривые $1-6$ ), с $\lambda_{0}=1>\lambda$ (черные кривые $1-6$ ) и с $\lambda_{0}=0,01<\lambda$ (штриховые кривые $1-6$ ). Значение $\gamma_{0}=0,9$ фиксировано, а номера кривых соответствуют шести значениям $\beta_{0}$ для каждой из трех моделей с разными временами объемной ретардации $\tau_{0}=1 / \lambda_{0}: \beta_{0}=1 ; 2 ; 3 ; 4 ; 5 ; 6$. С ростом $\beta_{0}$, то есть с убыванием объемных модулей $K_{\infty}=1 / \beta_{0}$ и $K=1 /\left(\beta_{0}-\gamma_{0}\right)$, график $v(t)$ смещается вниз и появляется интервал отрицательности КПД. Функции $v(t)$ всех трех моделей с одинаковым $\beta_{0}$ имеют одинаковые начальные значения $v(0)$ и асимптоты $v=v(\infty)$, $v(\infty)=-1+27\left(18+2 \beta_{0} / \beta\right)^{-1}$ (штриховые прямые $2^{\prime}, 6^{\prime}$ на рис. $1 a$ ) и могут менять знак (равновесные значения $v(\infty)$ отрицательны при $\beta_{0} / \beta>4,5$, то есть $G_{\infty} / K>1,5$ ), но в остальном ведут себя по-разному: при достаточно малом отношении $\tau_{0} / \tau<1$ $v(t)$ быстро убывает в окрестности нуля, а затем возрастает (черные кривые $1-6 \mathrm{c}$ $\left.\tau_{0} / \tau<0,1\right)$, при большом $\tau_{0} / \tau>1 v(t)$ возрастает вблизи нуля, а затем медленно убывает к асимптоте (штриховые кривые $2-6$ с $\tau_{0} / \tau=10$, их точки максимума за пределами рисунка), а при $\tau_{0}=\tau v(t)$ не имеет точек экстремума: убывает при малых $\beta_{0}<\beta_{*}, \beta_{*}=\beta \gamma_{0} / \gamma=9 / 5$ (голубая кривая 1 ) и возрастает при $\beta_{0}>\beta_{*}$ (а при $\beta_{0}=\beta_{*}$ $\xi(t)=$ const u $v(t)=$ const $)$. Таким образом, даже простейшая регулярная модель (23) с шестью параметрами и одноточечными спектрами сдвиговой и объемной релаксации и ретардации демонстрирует, насколько разнообразным может быть поведение КПД $v(t)$ и параметра $\xi(t)$ при нагружении (4), описываемое линейным ОС (1).

При мер 2. Рассмотрим фрактальную модель Максвелла со степенными ФП

$$
\Pi=B+A t^{u}, \quad \Pi_{0}=B_{0}+A_{0} t^{u}, \quad u, w \in(0,1), B, B_{0} \geq 0, A, A_{0}>0,
$$

(и непрерывными спектрами ретардации и релаксации). Ее мгновенный и длительные модули:

$$
\begin{gathered}
G=\frac{1}{2} \cdot \frac{2}{3} \Pi(0)^{-1}=\frac{1}{3} B^{-1}, \quad G_{\infty}=\frac{1}{2} \cdot \frac{2}{3} \Pi(\infty)^{-1}=0, \\
K=\frac{1}{\Pi_{0}(0)}=\frac{1}{B_{0}}, \quad K_{\infty}=\frac{1}{\Pi_{0}(\infty)}=0 .
\end{gathered}
$$

Для ФП (24) $Q=B t+A(u+1)^{-1} t^{u+1}, Q_{0}=B_{0} t+A_{0}(w+1)^{-1} t^{w+1}$ и согласно (17) $v(0)=$ 
$=-1+27\left[18+2 B_{0} / B\right]^{-1}, \xi(0)=(1 / 3) B_{0} / B$, а при $t \rightarrow \infty$ графики $v(t)$ обладают горизонтальными асимптотами: $\xi(\infty)=0, v(\infty)=0,5$ при $u>w, \xi(\infty)=+\infty, v(\infty)=-1$ при $u<w$ и $\xi(\infty)=(1 / 3) A_{0} / A, v(\infty)=-1+27\left[18+2 A_{0} / A\right]^{-1}$ при $u=w$ (в первых двух случаях асимптоты не зависят от параметров модели, и при больших временах моделируемый материал ведет себя как несжимаемый или как не меняющий форму). В силу (21)

$$
y(t)=\left(B_{0}+A_{0} t^{w}\right)\left[B t+A(u+1)^{-1} t^{u+1}\right]-\left[B_{0} t+A_{0}(w+1)^{-1} t^{w+1}\right]\left(B+A t^{u}\right),
$$

то есть

$$
y=A B_{0}\left[(u+1)^{-1}-1\right] t^{u+1}-A_{0} B\left[(w+1)^{-1}-1\right] t^{w+1}+A_{0} A\left[(u+1)^{-1}-(w+1)^{-1}\right] t^{u+w+1} .
$$

При $w=u \quad y=\left(A_{0} B-A B_{0}\right) u(u+1)^{-1} t^{u+1}$, и потому КПД - монотонная функция: при $A_{0} B>A B_{0} y(t)>0$ и КПД убывает на всем луче $t>0$, при $A_{0} B<A B_{0} y(t)<0$ и КПД возрастает на луче $t>0$, а при $A_{0} B=A B_{0} \mathrm{v}(t)=$ const. Отметим, что поперечная деформация (10) в случае $w=u$ имеет вид:

$$
\varepsilon_{\perp}=\frac{1}{18} b t\left[2 B_{0}-9 B+\left(2 A_{0}-9 A\right)(u+1)^{-1} t^{u}\right]
$$

и может быть не только монотонной (при условии $\left.C=\left(9 B-2 B_{0}\right) /\left(2 A_{0}-9 A\right) \leq 0\right)$, но и иметь точку максимума или минимума $\hat{t}=C^{1 / u}$, если $C>0(\hat{t}$ - точка максимума, если $9 B-2 B_{0}<0$ и $2 A_{0}-9 A_{0}<0$, и точка минимума, если $9 B-2 B_{0}>0$ и $2 A_{0}-9 A>$ $>0)$. Для модели (24) с $A_{0}=0$, то есть в случае пренебрежения объемной ползучестью, $y=-A B_{0} u(u+1)^{-1} t^{u+1}<0, \xi(t)$ убывает, а $v=0,5-3 B_{0}\left[18\left(B+A(u+1)^{-1} t^{u}\right)+\right.$ $\left.+2 B_{0}\right]^{-1}$ возрастает на всем луче $t>0$ и $v(\infty)=0,5$. Эти качественные свойства КПД моделей с $w=u$ или с $A_{0}=0$ в точности такие же, как и при ползучести [54]. Если $w \neq u$ и $A_{0} \neq 0$, то $y(t)$ и $\dot{v}(t)$ могут менять знак и КПД может быть немонотонным.

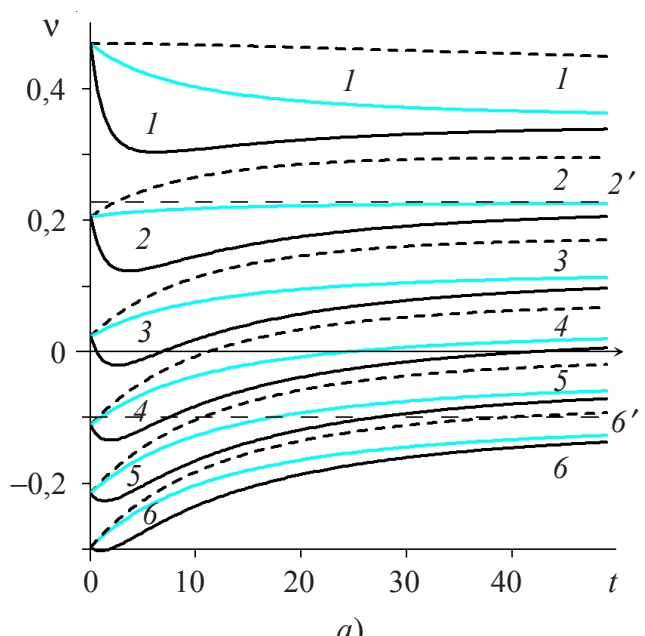

a)

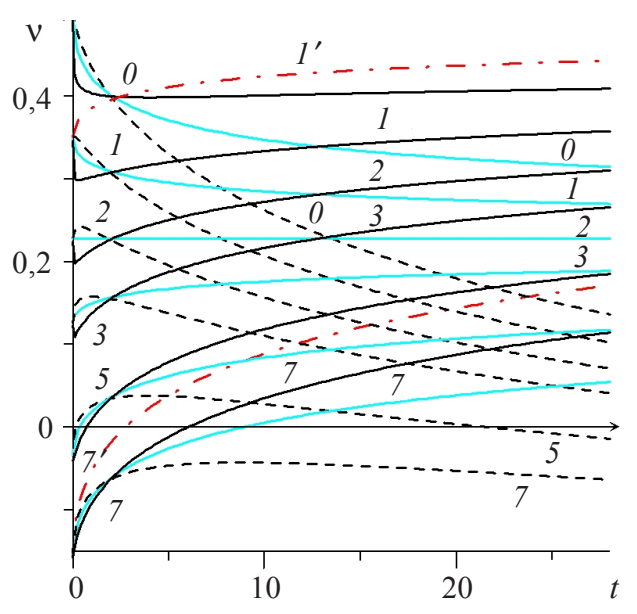

б)

Рис. 1. Графики КПД моделей семейства (23) с одинаковыми сдвиговыми ФП П $(t)$ и разными $\lambda_{0}$ и $\beta_{0}(a)$ и моделей $(24)$ с одинаковыми П $(t)$ и разными $w$ и $B_{0}(\sigma)$

На рис. 16 приведены графики КПД $v(t)$ при нагружении (4) трех моделей семейства (24) с одинаковыми сдвиговыми ФП П $(t)$ (с $u=0,5, A=0,5, B=1)$ и разными объемными ФП П $(t)$ : с $w=u=0,5$ (голубые кривые $0,1,2,3,5,7)$, с $w=0,2<u$ 
(черные кривые $0,1,2,3,5,7$ ) и с $w=0,8>u$ (штриховые кривые $0,1,2,3,5,7$ ). Параметр $A_{0}=1$ фиксирован, а номера кривых соответствуют разным значениям параметра $B_{0}=0 ; 1 ; 2 ; 3 ; 5 ; 7$ для каждой из трех моделей; с ростом $B_{0}$, то есть с убыванием мгновенного объемного модуля $K=1 / B_{0}$, график $v(t)$ смещается вниз). При каждом $B_{0}$ начальные значения $v(0)$ одинаковы у всех трех моделей (и убывают с ростом $B_{0}$ ), а горизонтальные асимптоты при $t \rightarrow \infty$ различны (и не зависят от $\left.B_{0}\right): \mathrm{v}(\infty)=0,5$ у всех моделей с $w<u, v(\infty)=-1$ в случае $w>u$ и $v(\infty)=-1+$ $+27\left[18+2 A_{0} / A\right]^{-1}=5 / 22$ при $w=u$. Асимптота голубых кривых $0-7 v=5 / 22$ совпадает с кривой (прямой) 2 , поскольку при $w=u$ и $B_{0}=2$ будет $A_{0} B=A B_{0}$ и $v(t)=$ const. Примечательны перемены знака и немонотонность $v(t)$ (у штриховой кривой $5-$ даже две перемены знака). Для сравнения приведены два графика $v(t)$ модели с линейно упругим изменением объема, то есть с $A_{0}=0, \Pi_{0}(t)=B_{0}$ (штрихпунктирные кривые $l^{\prime}$ и $7^{\prime}$ ): они монотонно возрастают и $v(\infty)=0,5$.

Для любой модели с $\Pi_{0}(t)=c=$ const $>0$ (без объемной ползучести) согласно (15), (14) и (21)

$$
\xi=\frac{1}{3} \frac{c t}{Q(t)}, \quad v=-1+\frac{27}{2}\left(9+\frac{c t}{Q(t)}\right)^{-1}, \quad y=c Q(t)-c t \Pi(t), \quad \dot{y}=-c t \dot{\Pi}(t)<0 .
$$

Поскольку $y(0)=0$ и убывает, то $y(t)<0$, и, следовательно, в силу (21) $\xi(t)$ убывает, a $v(t)$ возрастает на луче $t \geq 0$ (хотя $\varepsilon_{\perp}(t)$ может быть немонотонной - см. выше). Согласно (17)

$$
\begin{gathered}
\xi(0+)=\frac{1}{3} \cdot \frac{c}{\Pi(0)}, \quad \xi(\infty)=\frac{1}{3} \cdot \frac{c}{\Pi(\infty)}, \\
v(0+)=-1+\frac{27}{2} \cdot\left(9+\frac{c}{\Pi(0)}\right)^{-1}, \quad v(\infty)=-1+\frac{27}{2} \cdot\left(9+\frac{c}{\Pi(\infty)}\right)^{-1} .
\end{gathered}
$$

Если $\Pi(0)=0$, то $\xi(0+)=+\infty$ и $v(0+)=-1$, а если $\Pi(\infty)=\infty$, то $\xi(\infty)=0$ и $v(\infty)=0,5$. В частности, для модели (24) с $A_{0}=0$ имеем: $\xi(\infty)=0, v(\infty)=0,5, \xi(0+)=r / 3$, $v(0+)=-1+(27 / 2)(9+r)^{-1}$, где $r=B_{0} / B$, и $v(0+)<0$ при $9+r>27 / 2$, то есть $r>9 / 2$.

Примеры 1 и 2 показывают, в частности, что убывание КПД не обязательно связано с необратимыми объемными деформациями (как принято считать): все модели семейств (23) или (24) обеспечивают после снятия нагрузки полное восстановление объемных деформаций до нуля при $t \rightarrow \infty$ и обладают свойством затухания памяти, так как Пं( $\infty)=0[51,52,56]$.

\section{3. Сравнение поведения коэффициента Пуассона при нагружении (4) и при ползучести}

В условиях ползучести, то есть при одноосных нагружениях вида $\sigma_{11}(t)=\bar{\sigma} h(t)$, где $\bar{\sigma} \neq 0$, КПД и параметр вида деформированного состояния $\xi_{c}=\theta / \varepsilon$ выражаются формулами [54]:

$$
\begin{gathered}
v_{c}(t)=0,5-\frac{3 \Pi_{0}(t)}{18 \Pi(t)+2 \Pi_{0}(t)}=0,5-\frac{3 z_{c} \xi_{c}}{6+2 z_{c} \xi_{c}}=f\left(z_{c} \xi_{c}\right), \\
\xi_{c}(t)=\frac{1}{3} \cdot \frac{\bar{\sigma} \Pi_{0}(t)}{|\bar{\sigma}| \Pi(t)}=\frac{1}{3} \cdot z_{c} \frac{\Pi_{0}(t)}{\Pi(t)}, \quad z_{c}=\operatorname{sgn} \bar{\sigma}= \pm 1,
\end{gathered}
$$


где функция $f(x)$ определена в (14). Из формул (17) и (27) при $t \rightarrow 0+$ и $t \rightarrow+\infty$ следует, что $\xi(0+)=\xi_{c}(0+)$ и $\xi(+\infty)=\xi_{c}(+\infty)$, и потому $v(0+)=v_{c}(0+)$ и $v(+\infty)=$ $=\mathrm{v}_{c}(+\infty)$, то есть начальные и равновесные значения КПД при нагружении (4) такие же, как при ползучести.

Отношение $\xi_{c} / \xi=\Pi_{0}(t) Q(t) /\left[\Pi(t) Q_{0}(t)\right]\left(\right.$ и $\left.v_{c}(t) / v(t)\right)$ может быть и больше, и меньше единицы: неравенство $\xi_{c}(t)>\xi(t)$ в некоторый момент времени равносильно $y(t)>0$, где $y=\Pi_{0}(t) Q(t)-\Pi(t) Q_{0}(t)$ (см. (21)), а равенство $\xi_{c}(t)=\xi(t)$ равносильно $y(t)=0$, то есть условию (22). Поскольку функция $f(x)$ из (14) и (26) убывает при $x \geq 0$, то доказано следующее утверждение: неравенство $v_{c}(t)<v(t)$ на некотором интервале времени равносильно $y(t)>0$ (то есть условию $\dot{v}(t)<0$ в силу $(20)$ ), неравенство $v_{c}(t)>v(t)$ равносильно $y(t)<0$ (то есть $\dot{v}(t)>0$ ), а равенство $v_{c}(t)=v(t)$ равносильно условию (22), то есть достигается лишь в те моменты времени, которые совпадают с точками экстремума КПД (13) или лежат в интервалах его постоянства (в множестве нулей $y(t))$. Отметим, что $y$ моделей с упругой объемной деформацией (см. (25)) при любом $c>0$ всегда $y(t)<0$ и $\xi_{c} / \xi=t^{-1} Q(t) / \Pi(t)<1$ при $t>0$, то есть $\mathrm{v}_{c}(t)>\mathrm{v}(t)$.

На рис. 2 приведены для сравнения графики функций $\xi(t)$ и $\xi_{c}(t)$ (формулы (15) и (27)) при нагружении (4) с постоянной скоростью (сплошные кривые $1-4$ ) и при ползучести (штриховые кривые $1^{\prime}-4$ ) для шести моделей трех типов:

1) двух моделей вида (23) с $\beta=1, \gamma=0,5, \lambda=0,1, \beta_{0}=1, \gamma_{0}=0,9$ и $\lambda_{0}=1$ (кривые $\left.1,1^{\prime}\right)$ или $\lambda_{0}=0,1$ (кривые $2,2^{\prime}$ );

2) двух моделей (24) с $u=0,5, A=0,5, B=1, w=0,2, A_{0}=1$ и $B_{0}=0$ (красные кривые $3,3^{\prime}$ с $\left.\xi(0)=0\right)$ или $B_{0}=1$ (кривые $\left.4,4^{\prime}\right)$;

3) для модели с $\Pi_{0}(t)=c=$ const $>0$ (то есть модели (23) с $\gamma_{0}=0,9$ или модели (24) с $\left.A_{0}=0\right)$ при $c=B_{0}=0,5$ : синие кривые $5,5^{\prime}-$ при $B=1$ и кривые $6,6^{\prime}$ при $B=$ $=0($ когда $\xi(0+)=+\infty$ у модели (24)). У моделей (24) с $u>w \quad \xi(\infty)=0($ и $v(\infty)=0,5)$, а кривые $1,1^{\prime}, 2,2^{\prime}$ моделей (23) имеют общую асимптоту $\xi(\infty)=\beta_{0} /(3 \beta)=1 / 3$. Отношение $\xi_{c}(t) / \xi(t)$ может быть и больше, и меньше единицы (кривые $1-4$ и $l^{\prime}-$ $\left.4^{\prime}\right)$, а его пределы при $t \rightarrow 0+$ и $t \rightarrow+\infty$ равны единице.

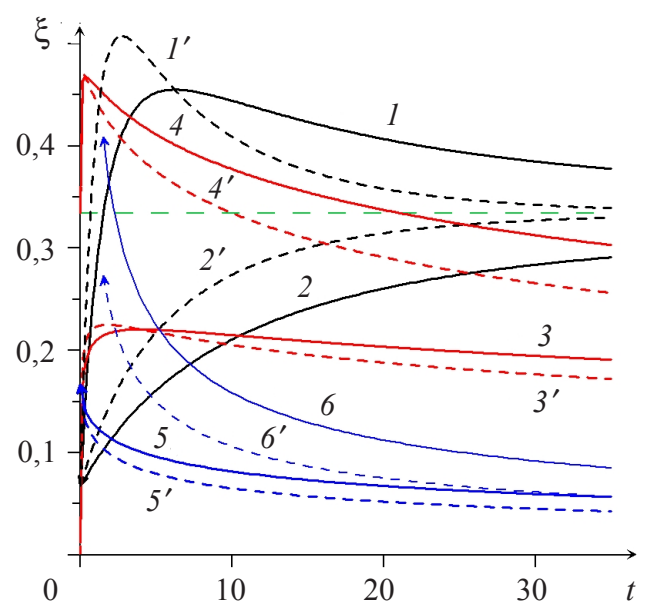

Рис 2. Графики $\xi(t)$ и $\xi_{c}(t)$ при нагружении (4) и при ползучести, порожденные моделями (23) и (24)

На рис. $3 a$ приведены графики КПД (13) при нагружении (4) с постоянной скоростью (кривые $0,1,2,3,5,7)$ и КПД (26) при ползучести (голубые штриховые 
кривые $0,1,2,3,5,7)$ для модели (24) с $u=0,5, A=0,5, B=1, A_{0}=1, w=0,2$ и $B_{0}=$ $=0 ; 1 ; 2 ; 3 ; 5 ; 7$. Для сравнения приведены два графика $v(t)$ модели с линейно упругим изменением объема, то есть с $A_{0}=0$ (штрихпунктирные кривые $l^{\prime}$ и $7^{\prime}$ ): они монотонно возрастают и $v(\infty)=0,5$. На рис. $3 \sigma-$ КПД при нагружении (4) (кривые $0,1,2,3,5,7)$ и при ползучести (голубые штриховые кривые $0,1,2,3,5,7)$ для модели (24) c $w=0,8$ и теми же значениями $B_{0}=0 ; 1 ; 2 ; 3 ; 5 ; 7$. Любой график КПД при ползучести быстрее стремится к равновесному значению (асимптоте) при $t \rightarrow \infty$, чем при нагружении (4): на рис. $3 a$ быстрее возрастает, а на рис. 36 - быстрее убывает.
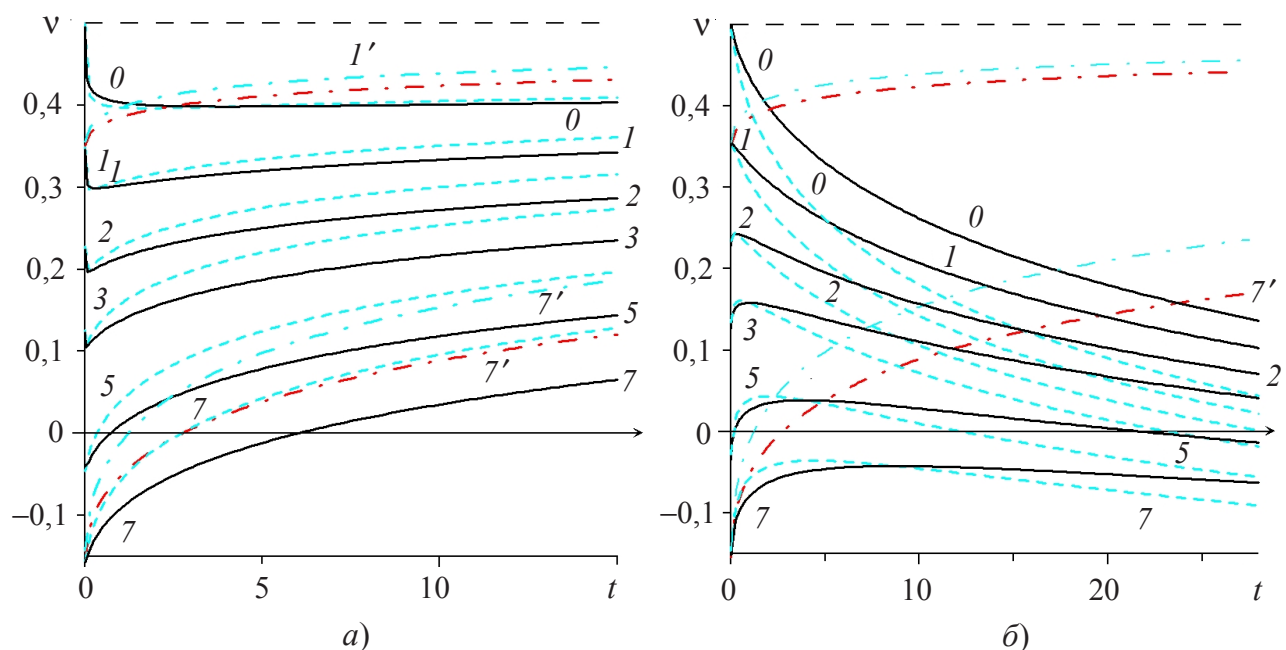

Рис 3. Графики КПД $v(t)$ при нагружении (4) и при ползучести, порожденные моделью (24) при $w=0,2$ (a) и при $w=0,8$ (б)

\section{Заключение}

Изучены возможности линейного ОС вязкоупругости (1) с двумя произвольными материальными функциями (ползучести) для изотропных нестареющих реономных материалов по описанию комплекса реологических эффектов, связанных с поведением коэффициента Пуассона (КПД) при одноосном нагружении (4) с постоянной скоростью. При минимальных (необходимых) ограничениях на функции ползучести (они предполагаются неотрицательными, возрастающими и выпуклыми вверх на полуоси времени) аналитически исследованы общие выражения (13) и (15) для КПД $v(t)$ при нагружении (4) и для параметра вида деформированного состояния $\xi(t)$ (равного отношению объемной деформации к интенсивности деформаций) через материальные функции и время. Доказано, что значения КПД $v(t)$ лежат в интервале $(-1 ; 0,5)$ при любом $t>0$ (универсальная оценка $(17)$ ), что функция $v(t)$ всегда имеет горизонтальную асимптоту при $t \rightarrow+\infty$, а начальная и равновесная величины КПД (16) могут принимать любые значения из отрезка $[-1 ; 0,5]$ (в зависимости от величины пределов отношения функций объемной и сдвиговой ползучести). Найдены критерии отрицательности КПД при нагружении (4), критерии его возрастания, убывания, постоянства и немонотонности. Обнаруженные свойства КПД (13) при нагружении (4) сопоставлены со свойствами КПД (26) в условиях ползучести (при постоянном напряжении), в частности, доказано, что каждый из них может превосходить другой на разных интервалах времени, что равенство этих 
КПД достигается только в те моменты времени, которые совпадают с точками экстремума КПД (13) или лежат в интервалах его постоянства (в множестве нулей функции $y(t)$ из (21)), а начальные и равновесные (предельные) значения КПД при нагружении (4) и КПД при ползучести всегда одинаковы.

Существенное отличие линейного ОС (1) от нелинейных ОС вязкоупругости независимость КПД от скорости нагружения и ее знака (как и от уровня напряжения при ползучести). Это свойство удобно проверять в испытаниях материалов по программам вида (4) и использовать как маркер границы области линейного поведения материалов, то есть индикатор неприменимости ОС (1) в случае обнаружения зависимости КПД от скорости нагружения (в некотором диапазоне скоростей и деформаций).

Таким образом, показано, что линейное ОС вязкоупругости (1) для нестареющих изотропных сред, пренебрегающее влиянием шаровой и девиаторной частей тензоров напряжений и деформаций друг на друга, способно качественно воспроизводить основные эффекты, связанные с поведением КПД при нагружении (4) (монотонность, немонотонность, знакопеременность, отрицательность КПД на некотором интервале времени, его стабилизацию с течением времени), за исключением зависимости от скорости нагружения.

Эффект отрицательности коэффициента Пуассона, развивающийся во времени, может быть использован, например, при создании самоукрепляющихся болтовых соединений, под нагрузкой превращающихся во фрикционные соединения за счет поперечного расширения болтов и их стеснения при растяжении (в частности, в условиях ползучести), и для улучшения прочностных и деформационных свойств материалов, плохо работающих на растяжение (цементы, бетоны, стекла и т.п.) за счет армирования ауксетичными волокнами и (микро)трубками (как направленного, так и хаотичного). Еще одно возможное перспективное направление - создание новых мягких магнитных композитов [61] (используемых в качестве разного рода актуаторов, клапанов, фиксаторов и уплотнителей), сочетающих управляемость свойств магнитным полем с возможностью выхода в режим «распухания» с отрицательным коэффициентом Пуассона.

\section{Список литературь}

1. Москвитин В.В. Сопротивление вязкоупругих материалов (применительно к зарядам ракетных двигателей на твердом топливе). М.: Наука, 1972. 328 с.

2. Айнбиндер С.Б., Тюнина Э.Л., Цируле К.И. Свойства полимеров в различных напряженных состояниях. М.: Химия, 1981. 232 с.

3. Гольдман А.Я. Объемная деформация пластмасс. Л.: Машиностроение, 1984. 232 с.

4. Гольдман А.Я. Прогнозирование деформационно-прочностных свойств полимерных и композиционных материалов. Л.: Химия, 1988. 272 с.

5. Брехова В.Д. Исследование коэффициента Пуассона при сжатии некоторых кристаллических полимеров постоянной нагрузкой. Механика полимеров. 1965. №4. С. 43-46.

6. Дзене И.Я., Путанс А.В. Коэффициент Пуассона при одномерной ползучести полиэтилена. Механика полимеров. 1967. №5. С. 947-949.

7. Дзене И.Я., Крегерс А.Ф., Вилкс У.К. Особенности процесса деформирования при ползучести и повторной ползучести полимеров в условиях одноосного растяжения. Часть 1. Механика полимеров. 1974. №3. С. 399-405.

8. Щербак В.В., Гольдман А.Я. Объемные изменения дисперсно наполненных композитов при испытании в условиях ползучести. Механика композитных материалов. 1982. №3. C. $549-552$. 
9. Калинников А.Е., Вахрушев А.В. О соотношении поперечной и продольной деформаций при одноосной ползучести разносопротивляющихся материалов. Механика композитных материалов. 1985. №2. С. 351-354.

10. Ozupek S., Becker E.B. Constitutive equations for solid propellants. Journal of Engineering Materials and Technology. 1997. Vol. 119. No 2. P. 125-132.

11. Okoli O.I., Smith G.F. The effect of strain rate and fibre content on the Poisson's ratio of glass/epoxy composites. Composite Structures. 2000. Vol. 48. No 1-3. P. 157-161. DOI: 10.1016/ S0263-8223(99)00089-6.

12. Hilton H.H. Implications and constraints of time-independent Poisson's ratios in linear isotropic and anisotropic viscoelasticity. Journal of Elasticity. 2001. Vol. 63. P. 221-251.

13. Tschoegl N.W., Knauss W.G., Emri I. Poisson's ratio in linear viscoelasticity - a critical review. Mechanics of Time-Dependent Materials. 2002. Vol. 6. Iss. 1. P. 3-51.

14. Arzoumanidis G.A., Liechti K.M. Linear viscoelastic property measurement and its significance for some nonlinear viscoelasticity models. Mechanics of Time-Dependent Materials. 2003. Vol. 7. Iss. 3-4. P. 209-250.

15. Cangemi L., Elkoun S., G'Sell C., Meimon Y. Volume strain changes of plasticized Poly(vinylidene fluoride) during tensile and creep tests. Journal of Applied Polymer Science. 2004. Vol. 91. P. 1784-1791.

16. Ломакин Е.В. Механика сред с зависящими от вида напряженного состояния свойствами. Физическая мезомеханика. 2007. Т. 10. №5. С. 41-52.

17. Pandini S., Pegoretti A. Time, temperature, and strain effects on viscoelastic Poisson's ratio of epoxy resins. Polymer Engineering and Science. 2008. Vol. 48. Iss. 7. P. 1434-1441.

18. O'Brien D.J., Sottos N.R., White S.R. Cure-dependent viscoelastic Poisson's ratio of epoxy. Experimental Mechanics. 2007. Vol. 47. Iss. 2. P. 237-249.

19. Быков Д.Л., Пелешко В.А. Определяющие соотношения деформирования и разрушения наполненных полимерных материалов в процессах преобладающего осевого растяжения в различных баротермических условиях. Изв. РАН. МТТ. 2008. №6. С. 40-65.

20. Grassia L., D'Amore A., Simon S.L. On the viscoelastic Poisson's ratio in amorphous polymers. Journal of Rheology. 2010. Vol. 54. P. 1009-1022. DOI: 10.1122/1.3473811.

21. Shekhar H., Sahasrabudhe A.D. Longitudinal strain dependent variation of Poissons ratio for HTPB based solid rocket propellants in uni-axial tensile testing. Propellants Explosives Pyrotechnics. 2011. Vol. 36. No 6. P. 558-563. DOI: 10.1002/prep.200900079.

22. Tscharnuter D., Jerabek M., Major·Z., Lang R.W. Time-dependent Poisson's ratio of polypropylene compounds for various strain histories. Mechanics of Time-Dependent Materials. 2011. Vol. 15. Iss. 1. P. 15-28. DOI: 10.1007/s11043-010-9121-x.

23. Kastner M., Obst M., Brummund J., Thielsch K., Ulbricht V. Inelastic material behavior of polymers - Experimental characterization, formulation and implementation of a material model. Mechanics of Materials. 2012. Vol. 52. P. 40-57.

24. Кожевникова М.Е. Характер изменения границы зоны пластичности и коэффициента Пуассона в зависимости от пластического разрыхления. Физическая мезомеханика. 2012. T. 15. №6. C. 59-66.

25. Cui H.R., Tang G.J., Shen Z.B. Study on viscoelastic Poisson's ratio of solid propellants using digital image correlation method. Propellants Explosives Pyrotechnics. 2016. Vol. 41. Iss. 5. P. 835-843. DOI: 10.1002/prep.201500313.

26. Lakes R. Foam structure with a negative Poisson's ratio. Science. 1987. Vol. 235. Iss. 4792. P. $1038-1040$.

27. Friis E.A., Lakes R.S., Park J.B. Negative Poisson's ratio polymeric and metallic materials. Journal of Materials Science. 1988. Vol. 23. Iss. 12. P. 4406-4414.

28. Caddock B.D., Evans K.E. Microporous materials with negative Poisson's ratios. I: Microstructure and mechanical properties. Journal of Physics D: Applied Physics. 1989. Vol. 22. No 12. P. 1877-1882.

29. Берлин Ал.Ал., Ротенбург Л., Басэрт Р. Особенности деформации неупорядоченных полимерных и неполимерных тел. Высокомолекулярные соединения. Б. 1992. Т. 34. №7. C. 6-32. 
30. Milton G.W. Composite materials with Poisson's ratios close to -1. Journal of the Mechanics and Physics of Solids. 1992. Vol. 40. Iss. 5. P. 1105-1137.

31. Alderson K.L., Evans K.E. The fabrication of microporous polyethylene having negative Poisson's ratio. Polymer. 1992. Vol. 33. Iss. 20. P. 4435-4438. DOI: 10.1016/0032-3861(92)90294-7.

32. Lakes R.S., Elms K. Indentability of conventional and negative Poisson's ratio foams. Journal Composite Materials. 1993. Vol. 27. Iss. 12. P. 1193-1202.

33. Chan N., Evans K.E. Indentation resilience of conventional and auxetic foams. Journal of Cellular Plastics. 1998. Vol. 34. Iss. 3. P. 231-260.

34. Alderson K.L., Fitzgerald A., Evans K.E. The strain dependent indentation resilience of auxetic microporous polyethylene. Journal of Materials Science. 2000. Vol. 35. Iss. 16. P. 4039-4047.

35. Конёк Д.А., Войцеховски К.В., Плескачевский Ю.М., Шилько С.В. Материалы с отрицательным коэффициентом Пуассона (обзор). Механ. композиц. матер. и конструкций. 2004. T. 10. №1. C. 35-69.

36. Liu Y., Hu H. A review on auxetic structures and polymeric materials. Scientific Research and Essays. 2010. Vol. 5. No 10. P. 1052-1063.

37. Greaves G.N., Greer A.L., Lakes R.S., Rouxel T. Poisson's ratio and modern materials. Nature Materials. 2011. Vol. 10. No 11. P. 823-837. DOI: 10.1038/NMAT3134.

38. Huang C., Chen L. Negative Poisson's ratio in modern functional materials. Advanced Materials. 2016. Vol. 28. No 37. P. 8079-8096. DOI: 10.1002/adma.201601363.

39. Volokh K.Yu. On arterial fiber dispersion and auxetic effect. Journal of Biomechanics. 2017. Vol. 61. P. 123-130.

40. Van der Varst P.G.Th., Kortsmit W.G. Notes on the lateral contraction of linear isotropic viscoelastic materials. Archive of Applied Mechanics. 1992. Vol. 62. Iss. 5. P. 338-346.

41. Hilton H.H., Sung Y. The significance of (an)isotropic viscoelastic Poisson ratio stress and time dependencies. International Journal of Solids and Structures. 1998. Vol. 35. No 23. P. 3081-3095. DOI: 10.1016/S0020-7683(97)00357-0.

42. Lakes R.S., Wineman A.S. On Poisson's ratio in linearly viscoelastic solids. Journal of Elasticity. 2006. Vol. 85. Iss 1. P. 45-63. DOI: 10.1007/s10659-006-9070-4.

43. Abudushalamu A., Vandamme M., Torrenti J.M., Benoit M. Theoretical and practical differences between creep and relaxation Poisson's ratios in linear viscoelasticity. Mechanics of TimeDependendent Materials. 2015. Vol. 19. Iss. 4. P. 537-555. DOI: 10.1007/s11043-015-9277-5.

44. Hilton H.H. Elastic and viscoelastic Poisson's ratios: The theoretical mechanics perspective. Materials Sciences and Applications. 2017. Vol. 8. No 4. P. 291-332. DOI: 10.4236/msa.2017.84021.

45. Айнбиндер С.Б., Алксне К.И., Тюнина Э.Л., Лака М.Г. Свойства полимеров при высоких давлениях. М.: Химия, 1973. 192 с.

46. Гольдштейн Р.В., Городцов В.А., Лисовенко Д.С. Изменчивость коэффициента Пуассона для гексагональных кристаллов под давлением. Труды МАИ. 2016. № 87. С. 1-22. http://trudymai.ru/published.php?ID=69526.

47. Векилов Ю.Х., Красильников О.М., Луговской А.В. Упругие свойства твердых тел при высоких давлениях. Успехи физических наук. 2015. Т. 185. №11. С. 1215-1224.

48. Хохлов А.В. Моделирование зависимости кривых ползучести при растяжении и коэффициента Пуассона реономных материалов от гидростатического давления с помощью нелинейно-наследственного соотношения Работнова. Механика композиционных материалов и конструкций. 2018. Т. 24. №3. С. 407-436.

49. Ильюшин А.А., Победря Б.Е. Основы математической теории термовязкоупругосmи. М.: Наука, 1970. 280 с.

50. Кристенсен Р. Введение в теорию вязкоупругости. М.: Мир, 1974. 338 с.

51. Хохлов А.В. Анализ общих свойств кривых ползучести при циклических ступенчатых нагружениях, порождаемых линейной теорией наследственности. Вестник Самарского гос. техн. ун-та. Сер. Физ.-мат. науки. 2017. Т. 21. №2. С. 326-361. DOI: 10.14498/vsgtu1533.

52. Хохлов А.В. Анализ свойств кривых ползучести с произвольной начальной стадией нагружения, порождаемых линейной теорией наследственности. Вестник Самарского гос. техн. ун-та. Сер. физ.-мат. науки. 2018. Т. 22. №1. С. 65-95. DOI: 10.14498/vsgtu1543.

53. Хохлов А.В. Двусторонние оценки для функции релаксации линейной теории наследственности через кривые релаксации при ramp-деформировании и методики ее иденти- 
фикации. Изв. РАН. МТТ. 2018. №3. C. 81-104. DOI: 10.7868/S0572329918030108.

54. Хохлов А.В. Особенности поведения поперечной деформации и коэффициента Пуассона изотропных реономных материалов при ползучести, описываемые линейной теорией вязкоупругости. Вестник ЮУрГУ. Сер. Математика. Механика. Физика. 2018. Т. 10. №4. C. 65-77. DOI: $10.14529 / \mathrm{mmph} 180408$.

55. Хохлов А.В. Анализ свойств кривых релаксации с начальной стадией ramp-деформирования, порождаемых нелинейной теорией наследственности Работнова. Механика композитных материалов. 2018. Т. 54. №4. С. 687-708. DOI: 10.1007/s11029-018-9757-1.

56. Хохлов А.В. Сравнительный анализ свойств кривых ползучести, порождаемых линейной и нелинейной теориями наследственности при ступенчатых нагружениях. Матем. физика и компьютер. моделир. 2018. Т. 21. №2. С. 27-51. DOI: 10.15688/mpcm.jvolsu.2018.2.3.

57. Хохлов А.В. О способности нелинейного определяющего соотношения Работнова для наследственных материалов моделировать диаграммы деформирования с падающим участком. Проблемы прочности и пластичности. 2018. Т. 80. №4. С. 477-493.

58. Хохлов А.В. Свойства семейства диаграмм деформирования, порождаемых нелинейным соотношением Работнова для вязкоупругопластичных материалов. Изв. РAH. MTT. 2019. №2. C. 29-47. DOI: 10.1134/S0572329919020077.

59. Работнов Ю.Н. Равновесие упругой среды с последействием. ПММ. 1948. Т. 12. №1. C. $53-62$.

60. Работнов Ю.Н. Ползучесть элементов конструкиий. М.: Наука, 1966. 752 с.

61. Molchanov V.S., Klepikov I.A., Razumovskaya I.V., Philippova O.E. Magnetically tunable viscoelastic response of soft magnetic nanocomposites with wormlike surfactant micellar matrix. Nanosystems: Physics, Chemistry, Mathematics. 2018. Vol. 9. No 3. P. 335-341. DOI: 10.17586/ 2220-8054-2018-9-3-335-341.

\section{References}

1. Moskvitin V.V. Soprotivlenie vyazkouprugikh materialov (primenitelno $k$ zaryadam raketnykh dvigateley na tverdom toplive) [Strength of Viscoelastic Materials]. Moscow. Nauka Publ. 1972. 328 p. (In Russian).

2. Aynbinder S.B., Tyunina E.L., Tsirule K.I. Svoystva polimerov v razlichnykh napryazhennykh sostoyaniyakh [Properties of Polymers under Various Stress States]. Moscow. Khimiya Publ. 1981. 232 p. (In Russian).

3. Goldman A.Ya. Ob'emnaya deformatsiya plastmass [Volumetric Deformation of Plastics]. Leningrad. Mashinostroenie Publ. 1984. 232 p. (In Russian).

4. Goldman A.Ya. Prognozirovanie deformatsionno-prochnostnykh svoystv polimernykh $i$ kompozitsionnykh materialov [Prediction of Deformation and Strength Properties of Polymeric and Composite Materials]. Leningrad. Khimiya Publ. 1988. 272 p. (In Russian).

5. Brekhova V.D. Investigation of the Poisson's ratio of certain crystalline polymers under a constant compressive load. Polymer Mech. 1965. Vol. 1. No 4. P. 23-24. https://doi.org/10.1007/ BF00858886.

6. Dzene I.Ya., Putans A.V. Poisson's ratio of polyethylene in one-dimensional creep. Polymer Mech. 1967. Vol. 3. No 5. P. 626-627. DOI: 10.1007/BF00859258.

7. Dzene I.Ya., Kregers A.F., Vilks U.K. Characteristic features of the deformation process on creep and secondary creep of polymers under conditions of uni-axial tension. Part I. Polymer Mechanics. 1974. Vol. 10. No 3. P. 337-342. DOI: 10.1007/BF00865585.

8. Shcherbak V.V., Goldman A.Ya. Ob'emnye izmeneniya dispersno napolnennykh kompozitov pri ispytanii v usloviyakh polzuchesti [Volume changes in dispersely filled composites under creep tests]. Mekhanika kompozitnykh materialov [Journal on Composite Mechanics and Design]. 1982. No 3. P. 549-552 (In Russian).

9. Kalinnikov A.E., Vakhrushev A.V. O sootnoshenii poperechnoy i prodolnoy deformatsiy pri odnoosnoy polzuchesti raznosoprotivlyayushchikhsya materialov [Relation between lateral and longitudinal strains in materials with tension-compression asymmetry under uniaxil creep]. Mekhanika kompozitnykh materialov [Journal on Composite Mechanics and Design]. 1985. No 2. P. 351-354 (In Russian). 
10. Ozupek S., Becker E.B. Constitutive equations for solid propellants. J. Engng. Mater. Technol. 1997. Vol. 119. No 2. P. 125-132.

11. Okoli O.I., Smith G.F. The effect of strain rate and fibre content on the Poisson's ratio of glass/epoxy composites. Composite Structures. 2000. Vol. 48. No 1-3. P. 157-161. DOI: 10.1016/ S0263-8223(99)00089-6.

12. Hilton H.H. Implications and constraints of time-independent Poisson's ratios in linear isotropic and anisotropic viscoelasticity. Journal of Elasticity. 2001. Vol. 63. P. 221-251.

13. Tschoegl N.W., Knauss W.G., Emri I. Poisson's ratio in linear viscoelasticity - a critical review. Mechanics of Time-Dependent Materials. 2002. Vol. 6. Iss. 1. P. 3-51.

14. Arzoumanidis G.A., Liechti K.M. Linear viscoelastic property measurement and its significance for some nonlinear viscoelasticity models. Mechanics of Time-Dependent Materials. 2003. Vol. 7. Iss. 3-4. P. 209-250.

15. Cangemi L., Elkoun S., G'Sell C., Meimon Y. Volume strain changes of plasticized Poly(vinylidene fluoride) during tensile and creep tests. Journal of Applied Polymer Science. 2004. Vol. 91. P. 1784-1791.

16. Lomakin E.V. Mechanics of media with stress-state dependent properties. Physical Mesomechanics. 2007. Vol. 10. No 5-6. P. 255-264.

17. Pandini S., Pegoretti A. Time, temperature, and strain effects on viscoelastic Poisson's ratio of epoxy resins. Polym. Eng. Sci. 2008. Vol. 48. Iss 7. P. 1434-1441.

18. O'Brien D.J., Sottos N.R., White S.R. Cure-dependent viscoelastic Poisson's ratio of epoxy. Exp. Mech. 2007. Vol. 47. P. 237-249.

19. Bykov D.L., Peleshko V.A. Constitutive relations for strain and failure of filled polymer materials in dominant axial tension processes under various barothermal conditions. Mechanics of Solids. 2008. Vol. 43. No 6. P. 870-891.

20. Grassia L., D'Amore A., Simon S.L. On the viscoelastic Poisson's ratio in amorphous polymers. J. Rheol. 2010. Vol. 54. P. 1009-1022. DOI: 10.1122/1.3473811.

21. Shekhar H., Sahasrabudhe A.D. Longitudinal strain dependent variation of Poissons ratio for HTPB based solid rocket propellants in uni-axial tensile testing. Propell. Explos. Pyrot. 2011. Vol. 36. No 6. P. 558-563. DOI: 10.1002/prep.200900079.

22. Tscharnuter D., Jerabek M., Major·Z., Lang R.W. Time-dependent Poisson's ratio of polypropylene compounds for various strain histories. Mech. Time-Depend. Mater. 2011. Vol. 15. Iss. 1. P. 15-28. DOI: 10.1007/s11043-010-9121-x.

23. Kastner M., Obst M., Brummund J., Thielsch K., Ulbricht V. Inelastic material behavior of polymers - Experimental characterization, formulation and implementation of a material model. Mech. Mater. 2012. Vol. 52. P. 40-57.

24. Kozhevnikova M.E. Plastic zone boundary and Poisson's ratio depending on plastic loosening. Physical Mesomechanics. 2013. Vol. 16. Iss. 2. P. 162-169.

25. Cui H.R., Tang G.J., Shen Z.B. Study on viscoelastic Poisson's ratio of solid propellants using digital image correlation method. Propell. Explos. Pyrot. 2016. Vol. 41. Iss 5. P. 835-843. DOI: $10.1002 /$ prep. 201500313.

26. Lakes R. Foam structure with a negative Poisson's ratio. Science. 1987. Vol. 235. Iss. 4792. P. $1038-1040$.

27. Friis E.A., Lakes R.S., Park J.B. Negative Poisson's ratio polymeric and metallic materials. J. Mater. Sci. 1988. Vol. 23. Iss. 12. P. 4406-4414.

28. Caddock B.D., Evans K.E. Microporous materials with negative Poisson's ratios. I: Microstructure and mechanical properties. Journal of Physics D: Applied Physics. 1989. Vol. 22. No 12. P. 1877-1882.

29. Berlin Al.Al., Rotenburg L., Basert R. Osobennosti deformatsii neuporiadochennykh polimernykh i nepolimernykh tel [Specific features of deformation of non-ordered polymeric and non-polymeric solids]. Vysokomolekuliarnye soedineniya. B [Polymer Science. Series B]. 1992. Vol. 34. No 7. P. 6-32 (In Russian).

30. Milton G.W. Composite materials with Poisson's ratios close to-1. J. Mech. Phys. Solids. 1992. Vol. 40. Iss. 5. P. 1105-1137.

31. Alderson K.L., Evans K.E. The fabrication of microporous polyethylene having negative Poisson's ratio. Polymer. 1992. Vol. 33. Iss. 20. P. 4435-4438. DOI: 10.1016/0032-3861(92)90294-7. 
32. Lakes R.S., Elms K. Indentability of conventional and negative Poisson's ratio foams. $J$. Composite Materials. 1993. Vol. 27. Iss. 12. P. 1193-1202.

33. Chan N., Evans K.E. Indentation resilience of conventional and auxetic foams. J. Cell. Plastics. 1998. Vol. 34. Iss. 3. P. 231-260.

34. Alderson K.L., Fitzgerald A., Evans K.E. The strain dependent indentation resilience of auxetic microporous polyethylene. J. Mater. Sci. 2000. Vol. 35. Iss. 16. P. 4039-4047.

35. Konek D.A., Voitsekhovski K.V., Pleskachevskii Iu.M., Shilko S.V. Materialy s otritsatelnym koeffitsientom Puassona (obzor) [Materials with negative Poisson's ratio. A review]. Mekhanika kompozitsionnykh materialov i konstruktsiy [Journal on Composite Mechanics and Design]. 2004. Vol. 10. No 1. P. 35-69 (In Russian).

36. Liu Y., Hu H. A review on auxetic structures and polymeric materials. Sci. Res. Essays. 2010. Vol. 5. No 10. P. 1052-1063.

37. Greaves G.N., Greer A.L., Lakes R.S., Rouxel T. Poisson's ratio and modern materials. Nat. Mater. 2011. Vol. 10. No 11. P. 823-837. DOI: 10.1038/NMAT3134.

38. Huang C., Chen L. Negative Poisson's ratio in modern functional materials. Adv. Mat. 2016. Vol. 28. No 37. P. 8079-8096. DOI: 10.1002/adma.201601363.

39. Volokh K.Yu. On arterial fiber dispersion and auxetic effect. Journal of Biomechanics. 2017. Vol. 61. P. 123-130.

40. Van der Varst P.G.Th., Kortsmit W.G. Notes on the lateral contraction of linear isotropic viscoelastic materials. Arch. Appl. Mech. 1992. Vol. 62. Iss. 5. P. 338-346.

41. Hilton H.H., Sung Y. The significance of (an)isotropic viscoelastic Poisson ratio stress and time dependencies. Int. J. Solids Struct. 1998. Vol. 35. No 23. P. 3081-3095. DOI: 10.1016/ S0020-7683(97)00357-0.

42. Lakes R.S., Wineman A.S. On Poisson's ratio in linearly viscoelastic solids. J. Elasticity. 2006. Vol. 85. Iss 1. P. 45-63. DOI: 10.1007/s10659-006-9070-4.

43. Abudushalamu A., Vandamme M., Torrenti J.M., Benoit M. Theoretical and practical differences between creep and relaxation Poisson's ratios in linear viscoelasticity. Mech. TimeDepend. Mater. 2015. Vol. 19. Iss. 4. P. 537-555. DOI: 10.1007/s11043-015-9277-5.

44. Hilton H.H. Elastic and viscoelastic Poisson's ratios: The Theoretical mechanics perspective. Materials Sciences and Applications. 2017. Vol. 8. No 4. P. 291-332. DOI: 10.4236/ msa.2017.84021.

45. Ainbinder S.B., Alksne K.I., Tiunina E.L., Laka M.G. Svoystva polimerov pri vysokikh davleniyakh [Properties of Polymers under High Pressure]. Moscow. Khimiya Publ. 1973. 192 p. (In Russian).

46. Goldshtein R.V., Gorodtsov V.A., Lisovenko D.S. Izmenchivost koeffitsienta Puassona dlya geksagonalnykh kristallov pod davleniem [Variability of Poisson's ratio for hexagonal crystals under pressure]. Trudy Moskovskogo aviatsionnogo instituta [Trudy MAI]. 2016. Vol. 87. P. 1-22. http://trudymai.ru/published.php?ID=69526 (In Russian).

47. Vekilov Yu.Kh., Krasilnikov O.M., Lugovskoy A.V. Elastic properties of solids at high pressure. Physics-Uspekhi. 2015. Vol. 58. No 11. P. 1106-1114.

48. Khokhlov A.V. Modelirovanie zavisimosti krivykh polzuchesti pri rastyazhenii i koeffitsienta Puassona reonomnykh materialov ot gidrostaticheskogo davleniya s pomoshchyu nelineynonasledstvennogo sootnosheniya Rabotnov [Simulation of hydrostatic pressure influence on creep curves and Poisson's ratio of rheonomic materials under tension using the Rabotnov non-linear hereditary relation]. Mekhanika kompozitsionnykh materialov i konstruktsiy [Mechanics of Composite Materials and Structures]. 2018. Vol. 24. No 3. P. 407-436 (In Russian).

49. Ilyushin A.A., Pobedrya B.E. Osnovy matematicheskoy teorii termovyazkouprugosti [Fundamentals of the Mathematical Theory of Thermo-Viscoelasticity]. Moscow. Nauka Publ. 1970. 280 p. (In Russian). $256 \mathrm{p}$.

50. Cristensen R.M. Theory of Viscoelasticity. An Introduction. N.-Y., L. Acad. Press. 1971.

51. Khokhlov A.V. Analiz obshchikh svoystv krivykh polzuchesti pri tsiklicheskikh stupenchatykh nagruzheniyakh, porozhdaemykh lineynoy teoriey nasledstvennosti [Analysis of creep curves produced by the linear viscoelasticity theory under cyclic stepwise loadings]. Vestnik Samarskogo gosudarstvennogo tekhnicheskogo universiteta. Seriya Fiziko-matematicheskie nauki [Journal of 
Samara State Technical University. Ser. Physical and Mathematical Sciences]. 2017. Vol. 21. No 2. P. 326-361. DOI: 10.14498/vsgtu1533 (In Russian).

52. Khokhlov A.V. Analiz svoystv krivykh polzuchesti s proizvolnoy nachalnoy stadiey nagruzheniya, porozhdaemykh lineynoy teoriey nasledstvennosti [Analysis of properties of creep curves generated by the linear viscoelasticity theory under arbitrary loading programs at initial stage]. Vestnik Samarskogo gosudarstvennogo tekhnicheskogo universiteta. Seriya Fiziko-matematicheskie nauki [Journal of Samara State Technical University. Ser. Physical and Mathematical Sciences]. 2018. Vol. 22. No 1. P. 65-95. DOI: 10.14498/vsgtu1543 (In Russian).

53. Khokhlov A.V. Two-sided estimates for the relaxation function of the linear theory of heredity via the relaxation curves during the ramp-deformation and the methodology of identification. Mechanics of Solids. 2018. Vol. 53. No 3. P. 307-328. DOI: 10.3103/S0025654418070105.

54. Khokhlov A.V. Osobennosti povedeniya poperechnoy deformatsii i koeffitsienta Puassona izotropnykh reonomnykh materialov pri polzuchesti, opisyvaemye lineynoy teoriey vyazkouprugosti [Behavior types and features of lateral strain and Poisson's ratio of isotropic rheonomous materials under creep conditions described by the linear theory of viscoelasticity]. Vestnik Yuzhnouralskogo gosudarstvennogo universiteta. Seriya Matematika. Mekhanika. Fizika [Bulletin of South Ural State University. Series of Mathematics. Mechanics. Physics]. 2018. Vol. 10. No 4. P. 65-77. DOI: 10.14529/mmph180408 (In Russian).

55. Khokhlov A.V. Analysis of properties of ramp stress relaxation curves produced by the Rabotnov non-linear hereditary theory. Mechanics of Composite Materials. 2018. Vol. 54. No 4. P. 473-486. DOI: 10.1007/s11029-018-9757-1.

56. Khokhlov A.V. Sravnitelnyy analiz svoystv krivykh polzuchesti, porozhdaemykh lineynoy i nelineynoy teoriyami nasledstvennosti pri stupenchatykh nagruzheniyakh [Comparative analysis of properties of creep curves produced by the Boltzmann - Volterra linear viscoelasticity relation and the Rabotnov quasi-linear relation under multi-step loadings]. Matematicheskaya fizika $i$ kompyuternoe modeliroranie [Mathematical Physics and Computer Simulation]. 2018. Vol. 21. No 2. P. 27-51. DOI: 10.15688/mpcm.jvolsu.2018.2.3 (In Russian).

57. Khokhlov A.V. O sposobnosti nelineynogo opredelyayushchego sootnosheniya Rabotnova dlya nasledstvennykh materialov modelirovat diagrammy deformirovaniya s padayushchim uchastkom [On the ability of the Rabotnov non-linear relation for viscoelastic materials to simulate stress-strain curves with a decreasing segment]. Problemy prochnosti i plastichnosti [Problems of Strength and Plasticity]. 2018. Vol. 80. No 4. P. 477-493. DOI: 10.32326/1814-9146-2018-80-4477-493 (In Russian).

58. Khokhlov A.V. Svoystva semeystva diagramm deformirovaniya, porozhdaemykh nelineynym sootnosheniem Rabotnova dlya vyazkouprugoplastichnykh materialov [Properties of stressstrain curves family generated by the Rabotnov non-linear relation for viscoelastic materials]. Izvestiya RAN. Mekhanika tverdogo tela [Mechanics of Solids]. 2019. No 2. P. 29-47. DOI: 10.1134/ S0572329919020077 (In Russian).

59. Rabotnov Yu.N. Ravnovesie uprugoy sredy s posledeystviem [Equilibrium of elastic hereditary medium]. Prikladnaya matematika i mekhanika [Journal of Applied Mathematics and Mechanics]. 1948. Vol. 12. No 1. P. 53-62 (In Russian).

60. Rabotnov Yu.N. Polzuchest elementov konstruktsiy [Creep Problems in Structural Members]. Moscow. Nauka Publ. 1966. 752 p. (In Russian).

61. Molchanov V.S., Klepikov I.A, Razumovskaya I.V., Philippova O.E. Magnetically tunable viscoelastic response of soft magnetic nanocomposites with wormlike surfactant micellar matrix. Nanosystems: Physics, Chemistry, Mathematics. 2018. Vol. 9. No 3. P. 335-341. DOI: 10.17586/ 2220-8054-2018-9-3-335-341. 


\title{
NON-MONOTONICITY, SIGN CHANGES AND OTHER FEATURES OF POISSON'S RATIO EVOLUTION FOR ISOTROPIC LINEAR VISCOELASTIC MATERIALS UNDER TENSION AT CONSTANT STRESS RATES
}

\author{
Khokhlov A.V.
}

Institute of Mechanics, Lomonosov Moscow State University, Moscow, Russian Federation

We study analytically the Boltzmann - Volterra linear constitutive equation for isotropic non-aging viscoelastic media in order to elucidate its capabilities to provide a qualitative simulation of rheological phenomena related to different types of evolution of triaxial strain state and of the lateral contraction ratio (the Poisson ratio) observed in uni-axial tests of viscoelastic materials under tension or compression at constant stress rate. In particular, we consider such effects as increasing, decreasing or non-monotone dependences of lateral strain and Poisson's ratio on time, sign changes and negativity of Poisson's ratio (auxeticity effect) and its stabilization at large times. The viscoelasticity equation implies that the hydrostatic and deviatoric parts of stress and strain tensors don't depend on each other. It is governed by two material functions of a positive real argument (that is shear and bulk creep compliances).

Assuming both creep compliances are arbitrary positive, differentiable, increasing and convex up functions on time semi-axis, we analyze general expressions for the Poisson ratio and strain triaxiality ratio (which is equal to volumetric strain divided by deviatoric strain) generated by the viscoelasticity relation under uni-axial tension or compression. We investigate qualitative properties and peculiarities of their evolution in time and their dependences on material functions characteristics. We obtain the universal accurate two-sided bound for the Poisson ratio range and criteria for the Poisson ratio increase or decrease and for extrema existence. We derive necessary and sufficient restrictions on shear and bulk creep compliances providing sign changes of the Poisson ratio and negative values of Poisson's ratio on some interval of time. The properties of the Poisson ratio under tension at constant stress rates found in the study we compare to properties the Poisson ratio evolution under constant stress (in virtual creep tests) and illustrate them using popular classical and fractal models with shear and bulk creep functions each one controlled by three parameters. The analysis carried out let us to conclude that the linear viscoelasticity theory (supplied with common creep functions which are non-exotic from any point of view) is able to simulate qualitatively the main effects associated with different types of the Poisson ratio evolution under tension or compression at constant stress rate except for dependence of Poisson's ratio on stress rate. It is proved that the linear theory can reproduce increasing, decreasing or non-monotone and convex up or down dependences of lateral strain and Poisson's ratio on time and it can provide existence of minimum, maximum or inflection points and sign changes from minus to plus and vice versa and asymptotic stabilization at large times.

Keywords: viscoelasticity, volumetric creep, tensile tests at constant stress rates, lateral contraction ratio, sign changes and non-monotonicity of Poisson's ratio, viscoelastic auxetics, evolution of auxetic behavior, magnetic composites, indicators of linear range limits. 